This item was submitted to Loughborough's Research Repository by the author.

Items in Figshare are protected by copyright, with all rights reserved, unless otherwise indicated.

\title{
Spectral structures of the generalized companion form and applications
}

PLEASE CITE THE PUBLISHED VERSION

LICENCE

CC BY-NC-ND 4.0

\section{REPOSITORY RECORD}

Pugh, Ashley Clive, and L. Tan. 2019. "Spectral Structures of the Generalized Companion Form and Applications”. figshare. https://hdl.handle.net/2134/884. 


\title{
Spectral structures of the generalised companion form and applications
}

\author{
A. C. Pugh, Liansheng Tan \\ Department of Mathematical Sciences, Loughborough University \\ Loughborough, Leicestershire, LE11 3TU, UK
}

\begin{abstract}
In this note, we investigate the relationship between the finite and infinite frequency structure of a regular polynomial matrix and that of a particular linearization, called the generalised companion matrix. A special resolvent decomposition of the regular polynomial matrix is proposed which is based on the Weierstrass canonical form of this generalised companion matrix and the solution of a regular PMD is thus formulated from this resolvent decomposition. Both the initial conditions of the pseudo state and the input are considered.
\end{abstract}

Corresponding Author: Dr. A. C. Pugh

Department of Mathematical Sciences

Loughborough University

Leicestershire, LE11 3TU, UK

Telephone:

+44 (0)1509223190

Telefax:

+44 (0)1509 211869

E-mail:

A.C.Pugh@lboro.ac.uk 


\section{Introduction}

Consider a regular polynomial matrix description (PMD) described by

$$
A(\rho) \beta(t)=B(\rho) u(t), \quad t \geq 0
$$

where $\rho:=d / d t$ is the differential operator, $A(\rho)=A_{q} \rho^{q}+A_{q-1} \rho^{q-1}+\cdots+A_{1} \rho+A_{0} \in$ $R[\rho]^{r \times r}, \operatorname{rank}_{R[\rho]} A(\rho)=r, A_{i} \in R^{r \times r}, i=0,1,2, \cdots, q, q \geq 1, B(\rho)=B_{l} \rho^{l}+B_{l-1} \rho^{l-1}+$ $\cdots+B_{1} \rho+B_{0} \in R[\rho]^{r \times m}, \quad B_{j} \in R^{r \times m}, j=0,1,2, \cdots, l, l \geq 0, \beta(t):[0,+\infty) \rightarrow R^{r}$ is the pseudo state of the PMDs, $u(t):[0,+\infty) \rightarrow R^{m}$ is an $p$ times piecewise continuously differentiable function called the input of the PMD.

A special case of (1) is the so-called generalized state space system (GSSS) in the following form

$$
(\rho E-A) x(t)=B u(t), \quad t \geq 0 .
$$

The solution of the above GSSS is well-known from the work of Gantmacher [1], Verghese [2], Campbell [3], Yip and Sincovec [4], Cobb [5]. Most of these results are based on Gantmacher's [1] analysis of the canonical form of the matrix pencil $s E-A$ called the Weierstrass canonical form. The convenience in actual computation can easily be seen, for only certain constant matrix transformations are necessary to bring the matrix pencil $s E-A$ to its Weierstrass canonical form, and from this the solution of the GSSS can be formulated.

More recently, many authors such as Gohberg et al. [6], Vardulakis and Fragulis [7], Vardulakis [8], and Fragulis [9] have discussed the solution of the regular PMD (1) . These solutions are all based on the resolvent decomposition [6], [8] of the regular polynomial matrix $A(s)$ which is formulated in terms of the finite Jordan pairs and the infinite Jordan pairs of $A(s)$. On the one hand, such treatments have the immediate advantage that they separate the system behaviour into the slow (smooth) response and the fast (impulsive) response which may provide a deep insight into the system structure. On the other hand, such treatments bring some inconvenience for the actual computation since the classical methods of determining the finite Jordan pairs or the infinite Jordan pairs have to transform the polynomial matrix $A(s)$ into its Smith-McMillan form or its SmithMcMillan form at infinity. However, such transformations are not stable in numerical computation terms, for they result in an extraordinarily large number of polynomial manipulations.

For simplicity, it is usual to assume that the initial conditions of the pseudo state $\beta(t)$ and the input $u(t)$ are all zero. However, in certain real cases, the initial conditions of the state might result from a random disturbance entering the system, and a feedback controller is thus called for. Since the precise value of the initial condition of the state is unpredictable and the control is likely to depend on those initial conditions of the state, it is somewhat stronger than necessary to assume that all the initial conditions of the state and the control are zero. This will be the position adopted in the sequel.

Since in (1) $B(\rho)$ contains the differential operator, not only the non-zero initial conditions of $\beta(t)$ but also the non-zero initial conditions of $u(t)$ should be considered, for both of them can create the impulsive modes as well as the slow response to the system.

In this note, a novel approach is presented to determine the complete solution of regular PMDs which takes into account not only the initial conditions on $\beta(t)$ but also the initial 
conditions on $u(t)$. One kind of linearization [6] of the regular polynomial matrix $A(s)$ is the so-called generalised companion matrix which is in fact a regular matrix pencil. Recently in [13], a realization approach was suggested that reduces high-order linear differential equations to the first-order system representations by using such generalized companion matrix. However, the complete structures of this companion matrix was not discussed in [13]. It is known that the Weierstrass canonical form of this matrix pencil can easily be obtained by certain constant matrix transformations. In this note certain properties of this companion form are established, and a special resolvent decomposition of $A(s)$ is proposed which is based on the Weierstrass canonical form. The solution of a regular PMD is then formulated from this resolvent decomposition. An obvious advantage of the approach adopted here is that it immediately avoids the polynomial matrix transformation necessary to obtain the finite and infinite Jordan pairs of $A(s)$, and only requires the constant matrix transformation to obtain the Weierstrass canonical form of the generalised companion form which is less sensitive than the former in computational terms. Since numerically efficient algorithms to generate the canonical form of a matrix pencil are well developed (see for example [16] [17]), the formula proposed here is more attractive in computational terms than the previously known results.

\section{The spectral structures of the generalised compan- ion form}

Let $A(s)=\sum_{i=0}^{q} A_{i} s^{i}$ be a regular $r \times r$ polynomial matrix. The generalized companion polynomial matrix $C_{A}(s)$ is defined as follows

$$
C_{A}(s):=\left[\begin{array}{ccccc}
I_{r} & 0 & \cdots & 0 & 0 \\
0 & I_{r} & \cdots & 0 & 0 \\
\vdots & \vdots & \ddots & \vdots & \vdots \\
0 & 0 & \cdots & I_{r} & 0 \\
0 & 0 & \cdots & 0 & A_{q}
\end{array}\right] s+\left[\begin{array}{ccccc}
0 & -I_{r} & 0 & \cdots & 0 \\
0 & 0 & -I_{r} & \cdots & 0 \\
\vdots & \vdots & \vdots & \ddots & \vdots \\
0 & 0 & 0 & \cdots & -I_{r} \\
A_{0} & A_{1} & A_{2} & \cdots & A_{q-1}
\end{array}\right] \text {, }
$$

where $I_{r} \in R^{r \times r}$ denotes the identity matrix. $C_{A}(s)$ is in fact a linearization of $A(s)$ for it satisfies

$$
E(s) C_{A}(s)=\left[\begin{array}{ll}
A(s) & 0_{r \times(q-1) r} \\
0_{(q-1) r \times r} & I_{(q-1) r \times(q-1) r}
\end{array}\right] F(s),
$$

where

$$
\begin{aligned}
& E(s):=\left[\begin{array}{ccccc}
A_{1}+s A_{2}+\cdots+s^{q-1} A_{q} & A_{2}+s A_{3}+\cdots+s^{q-2} A_{q} & \cdots & A_{q-1}+s A_{q} & I_{r} \\
-I_{r} & 0 & \cdots & 0 & 0 \\
0 & -I_{r} & \cdots & 0 & 0 \\
\vdots & \vdots & \ddots & \vdots & \vdots \\
0 & 0 & \cdots & -I_{r} & 0
\end{array}\right] \\
& F(s):=\left[\begin{array}{cccccc}
I_{r} & 0 & 0 & \cdots & 0 & 0 \\
-s I_{r} & I_{r} & 0 & \cdots & 0 & 0 \\
0 & -s I_{r} & I_{r} & \cdots & 0 & 0 \\
\vdots & \vdots & \vdots & \ddots & \vdots & \vdots \\
0 & 0 & 0 & \cdots & -s I_{r} & I_{r}
\end{array}\right]
\end{aligned}
$$


$C_{A}(s)$ is indeed a matrix pencil with a specially simple form. Such linearization originally appeared in [6], and was later used by [13]. Viewing the fact that $\operatorname{det} E(s)=1$ and $\operatorname{det} F(s)=1$, from (3) we deduce that $A(s)$ is regular as a polynomial matrix if and only if $C_{A}(s)$ is regular as a matrix pencil.

Proposition 1 (The Weierstrass canonical form of $C_{A}(s)$, [1]) For the regular matrix pencil $C_{A}(s)$, there exist nonsingular constant matrices $\mathrm{M}$ and $\mathrm{N}$ such that

$$
M C_{A}(s) N=\left[\begin{array}{cc}
s I_{n}-J & 0 \\
0 & s J_{\infty}-I_{\nu}
\end{array}\right]:=P(s)
$$

where $J$ is in Jordan canonical form with the finite zeros (Rosenbrock, [14]) of $C_{A}(s)$, $J_{\infty}:=$ block $\operatorname{diag}\left[J_{\infty 1}, J_{\infty 2}, \cdots, J_{\infty k}\right], J_{\infty i},(i=1, \cdots, k)$ are Jordan matrices with the size of $q_{i} \times q_{i}, q_{1} \leq q_{2} \leq \cdots \leq q_{k}, \nu=\sum_{i=1}^{k} q_{i}$, and $J_{\infty}$ is nilpotent with nilpotency $q_{k}$.

It is noted that the matrix $s I_{n}-J$ contains the finite elementary divisors of $C_{A}(s)$, and the matrix $s J_{\infty}-I_{\nu}$ contains the infinite elementary divisors [10] of $C_{A}(s)$. It is also noted in [6] that the matrix pencil $C_{A}(s)$ has the same finite elementary divisors as $A(s)$. For the relationship between the finite frequency structure of $A(s)$ and that of its companion matrix $C_{A}(s)$, the following result is obvious.

Proposition 2 The Smith-McMillan form of a matrix $\mathrm{H}(\mathrm{s})$ is denoted by $S_{H}^{C}(s)$. We have

$$
S_{C_{A}}^{C}(s)=\left[\begin{array}{ll}
I_{(q-1) r} & \\
& S_{A}^{C}(s)
\end{array}\right]=\left[\begin{array}{ll}
I_{\nu} & \\
& S_{\left(s I_{n}-J\right)}^{C}(s)
\end{array}\right]
$$

The above Proposition details the relationship between the finite frequency structure of $A(s)$ and that of its campanion form $C_{A}(s)$. The relationship between the infinite frequency structure of $A(s)$ and that of $C_{A}(s)$ is rather more obscure, as the transformation used in Proposition 1 together with the transformation of linearization do not preserve the infinite frequency structures of $C_{A}(s)$. Consequently there exists a significant difference between $S_{A}^{\infty}(s)$ and $S_{C_{A}}^{\infty}(s)$ as noted by [17]. The following important original result, on which the contribution of this paper is built, explains this difference.

Theorem 1 The Smith-McMillan form at infinity of a regular polynomial matrix $A(s)$ is $S_{A}^{\infty}(s)=\operatorname{diag}\left\{s^{q_{1}}, s^{q_{2}}, \cdots, s^{q_{k}}, 1 / s^{\hat{q}_{k+1}}, \cdots, 1 / s^{\hat{q}_{r}}\right\}$ iff the Jordan block matrices $J_{\infty i}(i=$ $1, \cdots, r-1)$ in the Weierstrass canonical form of its generalised companion matrix $C_{A}(s)$ are of dimensions $q_{1}-q_{2}, \cdots, q_{1}-q_{k}, q_{1}+\hat{q}_{k+1}, \cdots, q_{1}+\hat{q}_{r}$ respectively.

Proof If $A(s)=\sum_{i=0}^{q} A_{i} s^{i}$, its dual is defined [8] [10] as $D_{A}(w)=w^{q} \sum_{i=0}^{q} A_{i} \frac{1}{w^{i}}$. The infinite frequency structure of $A(s)$ is related to the finite frequency structure of its dual $D_{A}(w)$ at $w=0$ in the manner described in [10]. i.e., $S_{A}^{\infty}(s)=s^{q} S_{D_{A}}^{0}\left(\frac{1}{s}\right)$, where $S_{D_{A}}^{0}(w)$ denotes the local Smith form at $w=0$ of the dual of $A(s)$. Thus

$$
S_{A}^{\infty}(s)=\operatorname{diag}\left\{s^{q_{1}}, s^{q_{2}}, \cdots, s^{q_{k}}, 1 / s^{\hat{q}_{k+1}}, \cdots, 1 / s^{\hat{q}_{r}}\right\}
$$


where $q_{1}=q$, iff $S_{D_{A}}^{0}(w)=\operatorname{diag}\left\{1, w^{q_{1}-q_{2}}, \cdots, w^{q_{1}-q_{k}}, w^{\left(q_{1}+\hat{q}_{k+1}\right)}, \cdots, w^{\left(q_{1}+\hat{q}_{r}\right)}\right\}$. From (3) and (4), we have

$$
M E^{-1}(s)\left[\begin{array}{ll}
A(s) & 0_{r \times(q-1) r} \\
0_{(q-1) r \times r} & I_{(q-1) r \times(q-1) r}
\end{array}\right] F(s) N=\left[\begin{array}{cc}
s I_{n}-J & 0 \\
0 & s J_{\infty}-I_{\nu}
\end{array}\right] .
$$

Because $E(s)=s^{q-1} D_{E}\left(\frac{1}{s}\right), E^{-1}(s)=\frac{1}{s^{q-1}} D_{E}^{-1}\left(\frac{1}{s}\right)$, and $A(s)=s^{q} D_{A}\left(\frac{1}{s}\right)$, substituting into (5), we obtain

$$
M \frac{1}{s^{q-1}} D_{E}^{-1}\left(\frac{1}{s}\right)\left[\begin{array}{cc}
s^{q} D_{A}\left(\frac{1}{s}\right) & 0_{r \times(q-1) r} \\
0_{(q-1) r \times r} & I_{(q-1) r \times(q-1) r}
\end{array}\right] F(s) N=s\left[\begin{array}{cc}
I_{n}-\frac{1}{s} J & 0 \\
0 & J_{\infty}-\frac{1}{s} I_{\nu}
\end{array}\right],
$$

or alternatively since $w=\frac{1}{s}$

$$
M D_{E}^{-1}(w)\left[\begin{array}{cc}
D_{A}(w) & 0_{r \times(q-1) r} \\
0_{(q-1) r \times r} & w^{q} I_{(q-1) r \times(q-1) r}
\end{array}\right] F\left(\frac{1}{w}\right) N=\left[\begin{array}{cc}
I_{n}-w J & 0 \\
0 & J_{\infty}-w I_{\nu}
\end{array}\right] .
$$

Now we denote

$$
Q(w):=D_{E}^{-1}(w)\left[\begin{array}{cc}
D_{A}(w) & 0_{r \times(q-1) r} \\
0_{(q-1) r \times r} & w^{q} I_{(q-1) r \times(q-1) r}
\end{array}\right] F\left(\frac{1}{w}\right),
$$

and

$$
Q^{\prime}(w):=\left[\begin{array}{cc}
I_{n}-w J & 0 \\
0 & J_{\infty}-w I_{\nu}
\end{array}\right]
$$

Since M, N are both constant nonsingular matrices, we have

$$
S_{Q(w)}^{0}(w)=S_{Q^{\prime}(w)}^{0}(w) .
$$

By direct calculations, we find that

$$
\begin{aligned}
& D_{E}(w)= \\
& {\left[\begin{array}{ccccc}
A_{1} w^{q-1}+\cdots+A_{q} & A_{2} w^{q-1}+\cdots+A_{q} w & \cdots & A_{q-1} w^{q-1}+A_{q} w^{q-2} & w^{q-1} I_{r} \\
-w^{q-1} I_{r} & 0 & \cdots & 0 & 0 \\
0 & -w^{q-1} I_{r} & \cdots & 0 & 0 \\
\vdots & \vdots & \ddots & \vdots & \vdots \\
0 & 0 & \cdots & -w^{q-1} I_{r} & 0
\end{array}\right],} \\
& D_{E}^{-1}(w)= \\
& {\left[\begin{array}{ccccc}
0 & -\frac{1}{w^{q-1}} I_{r} & 0 & \cdots & 0 \\
0 & 0 & -\frac{1}{w^{q-1}} I_{r} & \cdots & 0 \\
0 & 0 & 0 & \cdots & 0 \\
\vdots & \vdots & \vdots & \ddots & \vdots \\
0 & 0 & 0 & \cdots & -\frac{1}{w^{q-1}} I_{r} \\
\frac{1}{w^{q-1}} I_{r} & \frac{A_{1} w^{q-1}+\cdots+A_{q}}{w^{2(q-1)}} & \frac{A_{2} w^{q-1}+\cdots+A_{q} w}{w^{2(q-1)}} & \cdots & \frac{A_{q-1} w^{q-1}+A_{q} w^{q-2}}{w^{2(q-1)}}
\end{array}\right]}
\end{aligned}
$$

Now

$$
F\left(\frac{1}{w}\right)=\left[\begin{array}{cccccc}
I_{r} & 0 & 0 & \cdots & 0 & 0 \\
-\frac{1}{w} I_{r} & I_{r} & 0 & \cdots & 0 & 0 \\
0 & -\frac{1}{w} I_{r} & I_{r} & \cdots & 0 & 0 \\
\vdots & \vdots & \vdots & \ddots & \vdots & \vdots \\
0 & 0 & 0 & \cdots & -\frac{1}{w} I_{r} & I_{r}
\end{array}\right]
$$


and so

$$
\begin{aligned}
Q(w)= & D_{E}^{-1}(w)\left[\begin{array}{ccc}
D_{A}(w) & 0 \\
0 & w^{q} I_{(q-1) r}
\end{array}\right] F\left(\frac{1}{w}\right) \\
= & {\left[\begin{array}{cccccc}
I_{r} & -w I_{r} & 0 & \cdots & 0 & 0 \\
0 & I_{r} & -w I_{r} & \cdots & 0 & 0 \\
0 & 0 & I_{r} & \cdots & 0 & 0 \\
\vdots & \vdots & \vdots & \ddots & \vdots & \vdots \\
0 & 0 & 0 & \cdots & I_{r} & -w I_{r} \\
A_{0} w & A_{1} w & A_{2} w & \cdots & A_{q-2} w & A_{q-1} w+A_{q}
\end{array}\right] . }
\end{aligned}
$$

It is seen that by unimodular transformations, $Q(w)$ can be brought to a diagonal block matrix $\operatorname{diag}\{\underbrace{I_{r}, \cdots, I_{r}}_{q-1}, D_{A}(w)\}$, subsequently $S_{Q(w)}^{0}(w)=\operatorname{diag}\left\{1, \cdots, 1, S_{D_{A}}^{0}(w)\right\}$, that is to say from (6)

$$
\left[\begin{array}{ll}
I_{(q-1) r} & \\
& S_{D_{A}}^{0}(w)
\end{array}\right]=\left[\begin{array}{ll}
I_{n} & \\
& S_{\left(-w I_{\nu}+J_{\infty}\right)}^{0}(w)
\end{array}\right] .
$$

Now by noticing that

$$
S_{\left(-w I_{\nu}+J_{\infty}\right)}^{0}(w)=\operatorname{diag}\{\underbrace{1, \cdots, 1}_{\nu-r+1}, w^{q_{1}-q_{2}}, \cdots, w^{q_{1}-q_{k}}, w^{\left(q_{1}+\hat{q}_{k+1}\right)}, \cdots, w^{\left(q_{1}+\hat{q}_{r}\right)}\},
$$

if and only if the associated Jordan block matrices $J_{\infty i}(i=1, \cdots, r-1)$ are of dimensions $q_{1}-q_{2}, \cdots, q_{1}-q_{k}, q_{1}+\hat{q}_{k+1}, \cdots, q_{1}+\hat{q}_{r}$ respectively, we finally obtain the required result.

Remark 1 If in Theorem 1 it happens that $q_{1}=q_{2}=\cdots=q_{h}$ for some $h \leq k$, then in the reconstruction of $S_{A}^{\infty}(s)$ from the Weierstrass canonical form of $C_{A}(s)$ it will be necessary to include $h$ polynomials of the form $s^{q_{1}}$. If the dimension of $A(s)$ is known (which will usually be the case) then $h$ is simply determined. In case the dimension of $A(s)$ is not known, this number $h$ can be determined from the formula

$$
\sharp\{\text { finite zeros }\}+\sharp\{\text { infinite zeros }\}+\sharp\{\text { infinite poles }\}=\operatorname{dim} C_{A}(s),
$$

where $\sharp$ denotes the number of such poles or zeros counted according to the algebraic multiplicities alone (i.e., taking no account of the geometric multiplicities).

Theorem 1 relates the infinite frequency structure of a polynomial matrix with the associated Weierstrass Canonical Matrix structure in a very natural way. Thus together with Propositions 1 and 2 one can immediately, from the Weierstrass canonical form of the generalized companion matrix, give characterizations of the finite and infinite frequency structure of the polynomial matrix $A(s)$. These results can be considered as the generalizations of the associated properties from the matrix pencil case to the polynomial matrix case. The above results are also interesting from the numerical computation point of view. They suggest an alternative way to find the finite and infinite frequency structure of a regular polynomial matrix. Since the finite and infinite frequency structure of a regular polynomial matrix are completely characterized by the Weierstrass canonical 
form of its companion matrix, the only thing we need to do is to transform the easily formed companion matrix (a regular matrix pencil) into its Weierstrass canonical form by some constant matrix transformation. Compared to the classical methods, which are by means of the polynomial matrix transformations, the method proposed here will be less sensitive to data perturbations and rounding errors.

\section{A new resolvent decomposition}

From Gohberg et al. [6] and Vardulakis [8], we can see that the resolvent decomposition of the regular polynomial matrix $A(s)$ plays a key role in formulating the solution of the regular PMD. The above linearization of $A(s)$ enables us to establish the following resolvent decomposition which is based on the Weierstrass canonical form of $C_{A}(s)$ and is different from that of Gohberg et al. [6] and Vardulakis [8].

Theorem 2 (A resolvent decomposition of $A(s)$ ) Let $A(s)=\sum_{i=0}^{q} A_{i} s^{i} \in R[s]^{r \times r}$ be regular and its companion matrix be $C_{A}(s)$. If the constant matrices $M \in R^{q r \times q r}$ and $N \in R^{q r \times q r}$ are those which reduce $C_{A}(s)$ into its Weierstrass canonical form (4) and M, $\mathrm{N}$ are partitioned as follows

$$
\begin{aligned}
M & :=\left[\begin{array}{ccc|c}
M_{11} & M_{12} & \cdots & M_{1 q} \\
M_{21} & M_{22} & \cdots & M_{2 q} \\
\vdots & \vdots & \vdots & \vdots \\
M_{q 1} & M_{q 2} & \cdots & M_{q q}
\end{array}\right], \text { where } M_{i j} \in R^{r \times r}, i=1, \cdots, q ; j=1, \cdots, q ; \\
N & :=\left[\begin{array}{cccc}
N_{11} & N_{12} & \cdots & N_{1 q} \\
\hline N_{21} & N_{22} & \cdots & N_{2 q} \\
\vdots & \vdots & \vdots & \vdots \\
N_{q 1} & N_{q 2} & \cdots & N_{q q}
\end{array}\right] \text {, where } N_{i j} \in R^{r \times r}, i=1, \cdots, q ; j=1, \cdots, q,
\end{aligned}
$$

then

$$
A^{-1}(s)=\left[N_{11}, N_{12}, \cdots, N_{1 q}\right]\left[\begin{array}{cc}
s I_{n}-J & 0 \\
0 & s J_{\infty}-I_{\nu}
\end{array}\right]^{-1}\left[\begin{array}{c}
M_{1 q} \\
M_{2 q} \\
\vdots \\
M_{q q}
\end{array}\right] .
$$

Proof Let $E_{q}(s)=A_{q}, E_{i-1}(s)=A_{i-1}+s E_{i}(s), i=q, \cdots, 2$. We observe that in (3)

$$
E(s)=\left[\begin{array}{ccccc}
E_{1}(s) & E_{2}(s) & \cdots & E_{q-1}(s) & I_{r} \\
-I_{r} & 0 & \cdots & 0 & 0 \\
0 & -I_{r} & \cdots & 0 & 0 \\
\vdots & \vdots & \ddots & \vdots & \vdots \\
0 & 0 & \cdots & -I_{r} & 0
\end{array}\right]
$$

SO

$$
E^{-1}(s)=\left[\begin{array}{ccccc}
0 & -I_{r} & 0 & \cdots & 0 \\
0 & 0 & -I_{r} & \cdots & 0 \\
\vdots & \vdots & \vdots & \ddots & \vdots \\
0 & 0 & 0 & \cdots & -I_{r} \\
I_{r} & E_{1}(s) & E_{2}(s) & \cdots & E_{q-1}(s)
\end{array}\right]
$$


From (3) and (4), we have

$$
M E^{-1}(s)\left[\begin{array}{ll}
A(s) & 0_{r \times(q-1) r} \\
0_{(q-1) r \times r} & I_{(q-1) r \times(q-1) r}
\end{array}\right] F(s) N=\left[\begin{array}{cc}
s I_{n}-J & 0 \\
0 & s J_{\infty}-I_{\nu}
\end{array}\right],
$$

SO

$$
\left[\begin{array}{cc}
A^{-1}(s) & 0 \\
0 & I_{(q-1) r}
\end{array}\right]=F(s) N\left[\begin{array}{cc}
s I_{n}-J & 0 \\
0 & s J_{\infty}-I_{\nu}
\end{array}\right]^{-1} M E^{-1}(s) .
$$

In fact

$$
\begin{gathered}
F(s) N=\left[\begin{array}{ccccc}
N_{11} & N_{12} & N_{13} & \cdots & N_{1 q} \\
\hline N_{21}-s N_{11} & N_{22}-s N_{12} & N_{23}-s N_{13} & \cdots & N_{2 q}-s N_{1 q} \\
\vdots & \vdots & \vdots & \vdots & \vdots \\
N_{q 1}-s N_{(q-1) 1} & N_{q 2}-s N_{(q-1) 2} & N_{q 3}-s N_{(q-1) 3} & \cdots & N_{q q}-s N_{(q-1) q}
\end{array}\right] \\
M E^{-1}(s)=\left[\begin{array}{c|ccccc}
M_{1 q} & M_{1 q} E_{1}(s)-M_{11} & M_{1 q} E_{2}(s)-M_{12} & \cdots & M_{1 q} E_{q-1}(s)-M_{1(q-1)} \\
M_{2 q} & M_{2 q} E_{1}(s)-M_{21} & M_{2 q} E_{2}(s)-M_{22} & \cdots & M_{2 q} E_{q-1}(s)-M_{2(q-1)} \\
\vdots & \vdots & \vdots & \vdots & \vdots \\
M_{q q} & M_{q q} E_{1}(s)-M_{q 1} & M_{q q} E_{2}(s)-M_{q 2} & \cdots & M_{q q} E_{q-1}(s)-M_{q(q-1)}
\end{array}\right] .
\end{gathered}
$$

According to the partition of the matrices in (8), we obtain the desired result.

The difference between the above resolvent decomposition of $A(s)$ and that of Gohberg et al. [6] and Vardulakis [8] lies in the fact that it is produced on the basis of the Weierstrass canonical form of the companion matrix $C_{A}(s)$ rather than on that of the finite and the infinite Jordan pairs of the original polynomial matrix. As stated previously, such treatment has an obvious advantage in computation. Although the dimension of $C_{A}(s)$ is larger than that of $A(s), C_{A}(s)$ is in fact sparse and has the special structure which facilities the computation. Computation of the finite and infinite Jordan pairs however is difficult. In the classical method, to find the finite and infinite Jordan matrices, one has to use unimodular matrix manipulations, numerical stability is thus lost because pivoting is based on the powers of the variable rather than on the coefficients as the method proposed here.

\section{Application to the solution of regular PMDs}

Consider the regular PMDs given by (1). Let the initial values of $u(t)$ and its $(l-1)$ derivatives at $t=0$ be $u(0), u^{(1)}(0) \cdots u^{(l-1)}(0)$ and the initial values of $\beta(t)$ and its $(q-1)$ derivatives at $t=0$ be $\beta(0), \beta^{(1)}(0) \cdots \beta^{(q-1)}(0)$. Taking the Laplace transformation of (1), we obtain

$$
A(s) \hat{\beta}(s)-\hat{\alpha}_{\beta}(s)=B(s) \hat{u}(s)-\hat{\alpha}_{u}(s)
$$

where $\hat{\beta}(s):=\int_{0}^{+\infty} \beta(t) e^{-s t} d t, \hat{u}(s):=\int_{0}^{+\infty} u(t) e^{-s t} d t$. Because of the Laplace-transformation rule

$$
\begin{aligned}
& L\left\{\frac{d^{i}}{d t^{i}} \beta(t)\right\}=s^{i} \hat{\beta}(s)-s^{i-1} \beta(0)-\cdots-s \beta^{(i-2)}(0)-\beta^{(i-1)}(0) ; \quad i=0,1, \cdots \\
& L\left\{\frac{d^{j}}{d t^{j}} u(t)\right\}=s^{j} \hat{u}(s)-s^{j-1} u(0)-\cdots-s u^{(j-2)}(0)-u^{(j-1)}(0) ; j=0,1, \cdots
\end{aligned}
$$


$\hat{\alpha}_{\beta}(s), \hat{\alpha}_{u}(s)$ can be written [11] as follows

$$
\begin{array}{r}
\hat{\alpha}_{\beta}(s)=\left[s^{q-1} I_{r}, s^{q-2} I_{r}, \cdots, s I_{r}, I_{r}\right]\left[\begin{array}{cccc}
A_{q} & 0 & \cdots & 0 \\
A_{q-1} & A_{q} & \cdots & 0 \\
\vdots & \vdots & \ddots & \vdots \\
A_{1} & A_{2} & \cdots & A_{q}
\end{array}\right]\left[\begin{array}{c}
\beta(0) \\
\beta^{(1)}(0) \\
\vdots \\
\beta^{(q-1)}(0)
\end{array}\right] \\
\hat{\alpha}_{u}(s)=\left[s^{l-1} I_{r}, s^{l-2} I_{r}, \cdots, s I_{r}, I_{r}\right]\left[\begin{array}{cccc}
B_{l} & 0 & \cdots & 0 \\
B_{l-1} & B_{l} & \cdots & 0 \\
\vdots & \vdots & \ddots & \vdots \\
B_{1} & B_{2} & \cdots & B_{l}
\end{array}\right]\left[\begin{array}{c}
u(0) \\
u^{(1)}(0) \\
\vdots \\
u^{(l-1)}(0)
\end{array}\right] .
\end{array}
$$

Now we repartition

$$
\hat{M}:=\left[\begin{array}{c}
M_{1 q} \\
M_{2 q} \\
\vdots \\
M_{q q}
\end{array}\right]:=\left[\begin{array}{c}
M_{n} \\
M_{\infty}
\end{array}\right], \text { where } M_{n} \in R^{n \times r}, M_{\infty} \in R^{\nu \times r},
$$

and

$$
\hat{N}:=\left[N_{11}, N_{12}, \cdots, N_{1 q}\right]:=\left[N_{n}, N_{\infty}\right] \text {, where } N_{n} \in R^{r \times n}, N_{\infty} \in R^{r \times \nu} .
$$

Note that

$$
n+\nu=q r
$$

According to Theorem 2, we have

$$
A^{-1}(s)=N_{\infty}\left(s J_{\infty}-I_{\nu}\right)^{-1} M_{\infty}+N_{n}\left(s I_{n}-J\right)^{-1} M_{n} .
$$

Similar to [8], we obtain

$$
A^{-1}(s) \hat{\alpha}_{\beta}(s)=\left[N_{n}, N_{\infty}\right]\left[\begin{array}{c|c}
s I_{n}-J & 0_{n, \nu} \\
\hline 0_{\nu, n} & s J_{\infty}-I_{\nu}
\end{array}\right]^{-1}\left[\begin{array}{c}
x_{s \beta}(0) \\
J_{\infty} x_{f \beta}(0)
\end{array}\right],
$$

where

$$
\begin{aligned}
{\left[\begin{array}{c}
x_{s \beta}(0) \\
J_{\infty} x_{f \beta}(0)
\end{array}\right]=} & {\left[\begin{array}{ccc|c}
J^{q-1} M_{n}, \cdots, M_{n} & 0_{n, q r} \\
\hline 0_{\nu, q r} & & J_{\infty} M_{\infty}, \cdots, J_{\infty}^{q} M_{\infty}
\end{array}\right] } \\
& \times\left[\begin{array}{cccc}
A_{q} & 0 & \cdots & 0 \\
A_{q-1} & A_{q} & \cdots & 0 \\
\vdots & \vdots & \ddots & \vdots \\
A_{1} & A_{2} & \cdots & A_{q} \\
\hline A_{0} & A_{1} & \cdots & A_{q-1} \\
0 & A_{0} & \cdots & A_{q-2} \\
\vdots & \vdots & \ddots & \vdots \\
0 & 0 & \cdots & A_{0}
\end{array}\right]\left[\begin{array}{c}
\beta(0) \\
\beta^{(1)}(0) \\
\vdots \\
\beta^{(q-1)}(0)
\end{array}\right] .
\end{aligned}
$$

Similarly,

$$
A^{-1}(s) \hat{\alpha}_{u}(s)=\left[N_{n}, N_{\infty}\right]\left[\begin{array}{c|c}
s I_{n}-J & 0_{n, \nu} \\
\hline 0_{\nu, n} & s J_{\infty}-I_{\nu}
\end{array}\right]^{-1}\left[\begin{array}{c}
x_{s u}(0) \\
J_{\infty} x_{f u}(0)
\end{array}\right],
$$


where

$$
\begin{aligned}
{\left[\begin{array}{c}
x_{s u}(0) \\
J_{\infty} x_{f u}(0)
\end{array}\right]=} & {\left[\begin{array}{cc|c}
J^{l-1} M_{n}, \cdots, M_{n} & 0_{n, l r} \\
\hline 0_{\nu, l r} & & J_{\infty} M_{\infty}, \cdots, J_{\infty}^{l} M_{\infty}
\end{array}\right] } \\
& \times\left[\begin{array}{cccc}
B_{l} & 0 & \cdots & 0 \\
B_{l-1} & B_{l} & \cdots & 0 \\
\vdots & \vdots & \ddots & \vdots \\
B_{1} & B_{2} & \cdots & B_{l} \\
\hline B_{0} & B_{1} & \cdots & B_{l-1} \\
0 & B_{0} & \cdots & B_{l-2} \\
\vdots & \vdots & \ddots & \vdots \\
0 & 0 & \cdots & B_{0}
\end{array}\right]\left[\begin{array}{c}
u(0) \\
u^{(1)}(0) \\
\vdots \\
u^{(l-1)}(0)
\end{array}\right] .
\end{aligned}
$$

We also obtain

$$
A^{-1}(s) B(s) \hat{u}(s)=\left[N_{n}, N_{\infty}\right] \Psi \Phi\left[\begin{array}{c}
I_{m} \\
s I_{m} \\
\vdots \\
s^{q_{k}-q+l} I_{m}
\end{array}\right] \hat{u}(s)+N_{n}\left[s I_{n}-J\right]^{-1} \Omega \hat{u}(s),
$$

where

$$
\begin{aligned}
& \Psi:=\left[\begin{array}{c|c}
J^{l-1} M_{n}, J^{l-2} M_{n}, \cdots, M_{n} & 0_{n,\left(q_{k}-q+1\right) r} \\
\hline 0_{\nu, l r} & M_{\infty}, J_{\infty} M_{\infty}, \cdots, J_{\infty}^{q_{k}-q} M_{\infty}
\end{array}\right] \\
& \Phi:=\left[\begin{array}{cccccccc}
B_{l} & 0 & \cdots & 0 & 0 & 0 & \cdots & 0 \\
B_{l-1} & B_{l} & \cdots & 0 & 0 & 0 & \cdots & 0 \\
\vdots & \vdots & \ddots & \vdots & \vdots & \vdots & \ddots & \vdots \\
B_{1} & B_{2} & \cdots & B_{l} & 0 & 0 & \cdots & 0 \\
\hline B_{0} & B_{1} & \cdots & B_{l-1} & B_{l} & 0 & \cdots & 0 \\
0 & B_{0} & \cdots & B_{l-2} & B_{l-1} & B_{l} & \cdots & 0 \\
\vdots & \vdots & \ddots & \vdots & \vdots & \ddots & \ddots & \vdots \\
0 & 0 & \cdots & B_{0} & B_{1} & \cdots & B_{l-1} & B_{l}
\end{array}\right] \\
& \Omega:=J^{l} M_{n} B_{l}+J^{l-1} M_{n} B_{l-1}+\cdots+J M_{n} B_{1}+M_{n} B_{0} .
\end{aligned}
$$

Now (9) can be written as

$$
\hat{\beta}(s)=A^{-1}(s) \hat{\alpha}_{\beta}(s)+A^{-1}(s) B(s) u(s)-A^{-1}(s) \hat{\alpha}_{u}(s)
$$

By taking the inverse Laplace transformation of the above, we finally obtain

Theorem 3 The complete solution of the regular PMDs (1) is

$$
\begin{aligned}
\beta(t)=\quad & N_{n} e^{J t} x_{s \beta}(0)-N_{n} e^{J t} x_{s u}(0) \\
& -N_{\infty} \sum_{i=1}^{q_{k}-q} \delta(t)^{(i-1)} J_{\infty}^{i-1}\left(J_{\infty} x_{f \beta}(0)\right)+N_{\infty} \sum_{i=1}^{q_{k}-q} \delta(t)^{(i-1)} J_{\infty}^{i-1}\left(J_{\infty} x_{f u}(0)\right) \\
& +\int_{0}^{t} N_{n} e^{J(t-\tau)} \Omega u(\tau) d \tau+\left[N_{n}, N_{\infty}\right] \Psi \Phi\left[\begin{array}{c}
u(t) \\
u(t)^{(1)} \\
\vdots \\
u(t)^{\left(q_{k}-q+l\right)}
\end{array}\right], t \geq 0 .
\end{aligned}
$$


From the above, it is clearly seen that the non-zero initial conditions of $\beta(t)$ and $u(t)$ both contribute to each of the the corresponding slow (smooth) zero input response

$$
N_{n} e^{J t} x_{s \beta}(0), \quad N_{n} e^{J t} x_{s u}(0)
$$

and the fast (impulse) response

$$
N_{\infty} \sum_{i=1}^{q_{k}-q} \delta(t)^{(i-1)} J_{\infty}^{i-1}\left(J_{\infty} x_{f \beta}(0)\right), \quad N_{\infty} \sum_{i=1}^{q_{k}-q} \delta(t)^{(i-1)} J_{\infty}^{i-1}\left(J_{\infty} x_{f u}(0)\right) .
$$

The above solution can be seen to be a natural generalization of that of GSSSs. However, in the case of the GSSSs, the initial conditions of $u(t)$ do not affect the solution structure, for wherein $B$ is independent of the differential operator.

\section{Conclusions}

In this paper, we have investigated the relationship between the finite and infinite frequency structure of a regular polynomial matrix and that of a simply determined companion matrix. It has been shown that in the Weierstrass canonical form of this generalised companion matrix, the finite Jordan block matrices determine the finite zeros of the original polynomial matrix and the dimensions of the infinite Jordan block matrices determine the infinite frequency structure of the original polynomial matrix and vice versa. A resolvent decomposition of $A(s)$ has been proposed based on the Weierstrass canonical form of the companion matrix which is easier to obtain than the finite Jordan pair and the infinite Jordan pair (Gohberg et al. [6] and Vardulakis [8]). Subsequently a solution procedure has been developed by using this resolvent decomposition.

The results of this paper enable one to give characterizations of the finite and infinite frequency structure of the polynomial matrix $A(s)$ simply from the Weierstrass canonical form of the generalized companion matrix. These results can be considered as the generalizations of the associated properties from the matrix pencil case to the polynomial matrix case. From the numerical computation point of view, these results suggest an alternative way of finding the finite and infinite frequency structure of a regular polynomial matrix. Since the finite and infinite frequency structure of a regular polynomial matrix are completely characterized by the Weierstrass canonical form of its companion matrix, the only thing we need to do is to transform the easily formed companion matrix (a regular matrix pencil) into its Weierstrass canonical form by some constant matrix transformation. Compared to the classical methods (Gohberg et al. [6] and Vardulakis [8]), which are by means of the polynomial matrix transformations, the method proposed here will be less sensitive to data perturbations and rounding errors. Based on this observation, the proposed solution procedure is thus more attractive in actual computation than the classical solution methods (Gohberg et al. [6] and Vardulakis [8]), for only certain constant matrix transformations are necessary to bring the matrix pencil $C_{A}(s)$ to its Weierstrass canonical form, and from this the solution of the regular PMD can be formulated.

\section{References}

[1] Gantmacher, F. R., The Theory of Matrices, Chelsea, New York, 1959 
[2] Verghese G. C., Infinite Frequency Behaviour in Dynamical Systems, Ph.D Dissertation, Department of Electronic Engineering, Stanford University, 1978.

[3] S.L. Campbell, Singular Systems of Differential Equations 1, London: Pitman , 1980.

[4] E., Yip and R., Sincovec, "Solvability, controllability and observability of continuous descriptor systems," IEEE Trans. Autom. Control vol. AC-26, No. 3, 702-707, 1981.

[5] D. Cobb, "On the solution of linear differential equations with singular coefficients," J. Differential Equations, vol. 46, pp. 310-323, 1982.

[6] Gohberg, I., Lancaster P. and Rodman I., Matrix Polynomials, Academic Press, New York, 1982.

[7] Vardulakis, A. I. G. and Fragulis G., "Infinite elementary divisors of polynomial matrices and impulsive solutions of linear homogeneous matrix differential equations", Circuit Systems and Signal Process, vol. 8, pp. 357-373, 1989.

[8] Vardulakis, A. I. G., Linear Multivariable Control, Algebraic Analysis and Synthesis Methods, John Willey and Sons, 1991.

[9] G. Fragulis, "A closed formula for the determination of the impulsive solutions of linear homogeneous matrix differential equations," IEEE Trans. Automat. Contr., vol. 38, no. 11, pp. 1688-1695, 1993.

[10] G. E. Hayton, A. C. Pugh and Fretwell P., "Infinite elementary divisors of a matrix polynomial and its implications", Int. J. Control, vol. 47, pp. 53-64, 1988.

[11] A. C. Pugh, "The McMillan degree of a polynomial system matrix", Int. J. Control, vol. 24, No. 1, 129-135, 1976.

[12] A. C. Pugh and Liansheng Tan, "On the Resolvent Decomposition of a Regular Polynomial Matrix and the Solution of Regular PMDs", Report Number A324, Department of Mathematical Sciences, Loughborough University, U. K., Feb., 1999.

[13] J. Rosenthal and J. M. Schumacher, "Realization by Inspection", IEEE Trans. on Auto. Control, vol. 42, no. 9, pp. 1257-1263, 1997.

[14] Rosenbrock, H. H., State-space and Multivariable Theory, London: Nelson-Wiley, 1970.

[15] F. Lewis, "A survey of linear singular systems", Circuits Systems Signal Process, vol. 5, no. 1, 1986.

[16] Jordan D. and Godbout L. F., "On the computation of the canonical pencil of a linear system," IEEE Trans. Automat. Contr., vol. 22, pp. 112-114,1977.

[17] Van Dooren P., “ The computation of Kronecker's canonical form of a singular pencil", Linear Algebra Appl., vol: 27, pp. 103-140, 1979.

[18] Van Dooren P., Verghese G. and Kailath T., " Properties of the system matrix of a generalized state-space system", in Proceedings of the 1978 IEEE Conference on Decision and Control, pp. 173-175. 
[19] Verghese, G.C., Lévy B.C. and Kailath, T., "A generalized state-space for singular system" IEEE Trans. on Automatic Control, AC-26, 811-831, 1981.

[20] Wolovich, W. A., Linear Multivariable Systems, Springer-Verlag, New York, 1974. 